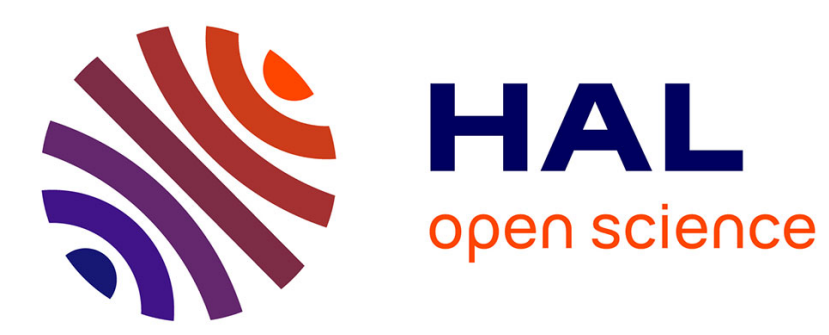

\title{
Pressure Boundary Conditions for Blood Flows
}

Kirill Gostaf, Olivier Pironneau

\section{- To cite this version:}

Kirill Gostaf, Olivier Pironneau. Pressure Boundary Conditions for Blood Flows. 2013. hal-00865671

\section{HAL Id: hal-00865671 \\ https: / hal.sorbonne-universite.fr/hal-00865671}

Submitted on 24 Sep 2013

HAL is a multi-disciplinary open access archive for the deposit and dissemination of scientific research documents, whether they are published or not. The documents may come from teaching and research institutions in France or abroad, or from public or private research centers.
L'archive ouverte pluridisciplinaire HAL, est destinée au dépôt et à la diffusion de documents scientifiques de niveau recherche, publiés ou non, émanant des établissements d'enseignement et de recherche français ou étrangers, des laboratoires publics ou privés. 


\title{
PRESSURE BOUNDARY CONDITIONS FOR BLOOD FLOWS
}

\author{
Kirill Pichon GOSTAF \\ Department of Mathematics, Simon Fraser University, Burnaby V5A 1S6, BC, Canada \\ Olivier Pironneau
}

LJLL-UPMC, Boite 187, Place Jussieu, 75252 PARIS cedex 5, France

\begin{abstract}
Simulations of blood flows in arteries require numerical solutions of fluid-structure interactions involving Navier-Stokes equations coupled with large displacement visco-elasticity for the vessels.

Among the various simplifications which have been proposed, the surface pressure model leads to a hierarchy of simpler models including one which involves only the pressure. The model exhibits fundamental frequencies which can be computed and compared with the pulse. Yet unconditionally stable time discretizations can be constructed by combining implicit time schemes with Galerkin-characteristic discretization of the convection terms in the NavierStokes equations. Such problems with prescribed pressure on the walls will be shown to be efficient and accurate as an approximation of the full fluid structure interaction problem.
\end{abstract}

1. Introduction. Computational Hemodynamics is a major research field with many applications to the human blood circulatory system - especially for heart diseases and aneurysms - leading to diagnostic tools and simulations of chirurgical treatments like stents (see [34]) and by-passes (see Thiriet[35]).

Since the pioneering work of Peskin [27] impressive progress has been made both on the methodological side, namely the treatment of moving walls, cardiac muscles etc, and on the numerical side for efficient and stable discretizations of these fluid structure interaction problems. Some are presented in [13]; mostly with methods on fixed domains after a change of variables (see [7, 24, 12]. For other methods like the fictitious domain and immersed boundaries methods the reader is referred to $[27,26,36]$ and[2], among others. Level sets has not been as popular but it can be used also as in [5].

In this paper we shall focus on aortic flows. Typically a large artery like the aorta has a radius of $1 \mathrm{~cm}$ and a length of 5 to 10 centimeters; the thickness of the aortic wall is around $0.1 \mathrm{~cm}$; the heart pulse is about $1 \mathrm{~Hz}$ and the pressure drop roughly $6 \mathrm{KPa}$. Knowing the density and viscosity this fixes a typical speed for the blood. At such speed the flow is Newtonian, incompressible with a Reynolds number of a few thousands.

2000 Mathematics Subject Classification. Primary: 91B28, 65L60; Secondary: 82B31.

Key words and phrases. Partial Differential Equations, Hemodynamics, numerical methods, Navier-Stokes, pressure boundary conditions.

This article is dedicated to Luc Tartar for his sixty-six birthday. 
The aorta is surrounded by organs so in all its generality the problem is very complex: large displacement visco-elasticity deformable solid with contacts on the surrounding organs and fluid-structure interaction (FSI) with a flow modeled by the Navier-Stokes equations in a moving domain.

2. Modeling the Aortic Wall: the Surface Pressure Model. The modeling and simulations of solids having large displacements and contacts is difficult but not impossible [16, 17]; rubber tires in particular are modeled as such; but it is computationally very expensive and even more so in the context of blood flow (see the computations of M. Vidrascu in [23].)

A hierarchy of approximations have been proposed. First, replace large displacement nonlinear elasticity by small displacements linear elasticity [6], then use shell models like Koiter's as in $[22,8]$ and finally assume that the displacement $\vec{d}$ is normal to the aortic wall ${ }^{1}$ :

$$
\vec{d}(x, t)=\eta(x, t) \vec{n}(x)
$$

In such case, as shown by Nobile \& Vergana [24] Koiter's model reduces to a scalar equation for $\eta$

$$
\rho^{s} h \partial_{t t} \eta-\nabla \cdot(\mathbf{T} \nabla \eta)-\nabla \cdot\left(\mathbf{C} \nabla \partial_{t} \eta\right)+a \partial_{t} \eta+b \eta=f^{s}, \eta, \partial_{t} \eta \text { given at } t=0
$$

on the mean position $\Sigma$ of the vessel's wall; $h$ denotes its average thickness and $\rho^{s}$ its density; $\mathbf{T}$ is the pre-stress tensor, needed because at rest the vessel is blown up by the blood like a balloon; $\mathbf{C}$ is a damping term, $a, b$ are viscoelastic terms and $f^{s}$ the external normal force, i.e. $-\sigma^{\mathbf{s}}{ }_{n n}$ the normal component of the normal stress at the surface of the solid.

The system is simplified further by assuming that the normal derivatives, $\partial_{n}$, dominate the tangential ones.

$$
\rho_{s} h \partial_{t t} \eta-\partial_{n}\left(T \partial_{n} \eta\right)-\partial_{n}\left(C \partial_{n t} \eta\right)+a \partial_{t} \eta+b \eta=f^{s}, \eta, \partial_{t} \eta \text { given at } t=0
$$

The final approximation is to assume that $[h, T, C, a]<<b$ which leads to the Surface Pressure Model

$$
-\sigma^{\mathbf{s}}{ }_{n n}=b \eta, \text { with } b=\frac{E h \pi}{A\left(1-\xi^{2}\right)}
$$

where $A$ is the vessel's cross section, $E$ the Young modulus, $\xi$ the Poisson coefficient. Some typical values (MKSA):

$$
E=3 M P a, \xi=0.3, A=\pi R^{2}, R=0.01, h=0.001, \Rightarrow b=3.310^{7} \mathrm{~ms}^{-2}
$$

3. Modeling Fluids with Given Boundary Pressure or Stress. For Newtonian incompressible fluids, pressure $p$ and velocity $\vec{u}$ are given by

$$
\rho^{f}\left(\frac{\partial \vec{u}}{\partial t}+\vec{u} \cdot \nabla \vec{u}\right)+\nabla \cdot \sigma^{\mathbf{f}}=0, \nabla \cdot \vec{u}=0,
$$

where $\rho^{f}$ is the density of the fluid, $\mu$ the viscosity and $\sigma^{f}=-p \mathbf{I}+\mu\left(\nabla u+\nabla u^{T}\right)$ is the stress tensor.

Many boundary conditions have been proposed for the artificial boundaries created by taking only a small portion of the blood circulatory system (see [37, 10, 12]).

\footnotetext{
${ }^{1}$ We use the vector notation $\vec{n}$ for the normal vector, for instance, to improve readability; when not ambiguous, we simply write $n$.
} 
In our problem the velocity and pressure are given at time $t=0$, the pressure is given on $\Gamma$ (the inlet and the outlet) and the normal stress is given on $\Sigma$ by the structure model for the compliant wall. Naturally $\Gamma \cup \Sigma=\partial \Omega$. As the inlet and outlet are artificial boundaries, we assume that $\Gamma$ is a union of plane surfaces (i.e. the two radii of curvature are infinite). Furthermore we assume that the flow is normal to the inlet and outlet; the Surface Pressure Model also implies normal velocity on $\Sigma$ :

$$
\left.u\right|_{t=0}=u^{0}, \vec{u} \times \vec{n}=0 \text { on } \partial \Omega,\left.p\right|_{\Gamma}=p_{\Gamma}, \sigma_{n n}^{f}=s \text { on } \Sigma
$$

A variational formulation is obtained by multiplying the first equation in (5) by $\hat{u}$ and the second by $\hat{p}$. Then for all $\hat{p}$ and all $\hat{u}$ such that $\hat{u} \times n=0$ on $\partial \Omega$ :

$$
\begin{aligned}
& \int_{\Omega}\left[\rho^{f}\left(\partial_{t} u \cdot+u \cdot \nabla u\right) \cdot \hat{u}+\frac{\mu}{2}\left(\nabla u+\nabla u^{T}\right):\left(\nabla \hat{u}+\nabla \hat{u}^{T}\right)-p \nabla \cdot \hat{u}-\hat{p} \nabla \cdot u\right] \\
& =\int_{\partial \Omega} \sigma_{n n}^{f} \hat{u}_{n}=\int_{\Sigma} s \hat{u}_{n}-\int_{\Gamma} p_{\Gamma} \hat{u}_{n}
\end{aligned}
$$

where $\hat{u}_{n}:=\hat{u} \cdot n$. Indeed when $\Gamma$ is flat, $u \times n=0, \nabla \cdot u=0, \Rightarrow \sigma_{n n}^{f}=-p$ because the tangential derivatives of $u$ are zero.

Such pressure boundary conditions were studied in [29, 1] (see also [28]). Existence and uniqueness may be proved as in the classical case following [15] and finite element approximations have been studied in [25] but there are some difficulties. First, because of the condition $u \times\left. n\right|_{\partial \Omega}=0$ one aught to use the curl-curl formulation:

$$
\int_{\Omega}\left[\left(\partial_{t} u \cdot+u \cdot \nabla u\right) \cdot \hat{u}+\mu \nabla \times u \cdot \nabla \times \hat{u}-p \nabla \cdot \hat{u}-\hat{p} \nabla \cdot u\right]=\int_{\Sigma} s \hat{u}_{n}-\int_{\Gamma} p_{\Gamma} \hat{u}_{n}, \forall \hat{u}, q
$$

Recovery of the strong form of the Navier-Stokes equations when regularity allows makes use of the formula (see [3, 4]): for all $u, v \in H^{2}(\Omega)$ with either $u \times n=$ $v \times n=0$ or $u \cdot n=v \cdot n=0$ on $\partial \Omega$,

$$
\begin{aligned}
& \int_{\Omega} {[\nabla \times u \cdot \nabla \times v+\nabla \cdot u \nabla \cdot v]=\int_{\Omega} \nabla u: \nabla v+b(u, v) } \\
&=\int_{\Omega}\left[\frac{1}{2}\left(\nabla u+\nabla u^{T}\right):\left(\nabla v+\nabla v^{T}\right)-\nabla \cdot u \nabla \cdot v\right]+2 b(u, v)
\end{aligned}
$$

with $b(u, v)=\int_{\partial \Omega}\left[\left(\left(u-u_{n} n\right) \cdot \nabla n\right) \cdot\left(v-v_{n} n\right)+u_{n}(\nabla \cdot n) v_{n}\right]$. Furthermore if $\partial \Omega$ is piecewise plane, then

$$
\begin{aligned}
\int_{\Omega}[\nabla \times u \cdot \nabla \times v & +\nabla \cdot u \nabla \cdot v]=\int_{\Omega} \nabla u: \nabla v \\
& =\int_{\Omega}\left[\frac{1}{2}\left(\nabla u+\nabla u^{T}\right):\left(\nabla v+\nabla v^{T}\right)-\nabla \cdot u \nabla \cdot v\right]
\end{aligned}
$$

\section{The Fluid - Structure Interaction Problem.}

4.1. Variational Form of the Model for $[\vec{u}, p, \eta]$. Coupling the two systems of equations (3),(7) and writing the continuity of the velocities at the interface $\Sigma$, namely $\vec{u}=\partial_{t} \vec{d} \approx \vec{n} \partial_{t} \eta$ leads to find $[\vec{u}, p, \eta]$ with $u \times n=0,\left.\eta\right|_{\Gamma}=0$ and

$$
\begin{aligned}
& \int_{\Omega}\left[\rho^{f}\left(\partial_{t} u \cdot+u \cdot \nabla u\right) \cdot \hat{u}+\frac{\mu}{2}\left(\nabla u+\nabla u^{T}\right):\left(\nabla \hat{u}+\nabla \hat{u}^{T}\right)-p \nabla \cdot \hat{u}-\hat{p} \nabla \cdot u\right] \\
& +\int_{\Sigma} b\left[\eta \hat{u}_{n}+\hat{\eta}\left(u_{n}-\partial_{t} \eta\right)\right]=-\int_{\Gamma} p_{\Gamma} \hat{u}_{n}, \forall \hat{u}, \hat{p}, \hat{\eta} \text { with } \hat{u} \times n=0,\left.\hat{\eta}\right|_{\Gamma}=0 .
\end{aligned}
$$


It must be noted that $\Omega$ and its boundary are functions of time and must be updated by the displacement $\vec{d}$ as in [11]. As it is unfeasible to move $\Gamma$ we assume that $\left.\eta\right|_{\Gamma}=0$.

An energy conservation identity is obtained by letting $\hat{u}=u, \hat{p}=-p, \hat{\eta}=-\eta$ :

$$
\int_{\Omega}\left[\rho^{f} \partial_{t} u \cdot u+\frac{\mu}{2}\left|\nabla u+\nabla u^{T}\right|^{2}+\int_{\partial \Omega}\left[\frac{u_{n}}{2} \rho^{f}|u|^{2}+b \eta \partial_{t} \eta\right]=-\int_{\Gamma} p_{\Gamma} u_{n}\right.
$$

Making use of the identity

$$
\partial_{t} \int_{\Omega(t)} \frac{|u|^{2}}{2}=\int_{\Omega(t)} \partial_{t} u \cdot u+\int_{\partial \Omega(t)} \frac{u_{n}}{2}|u|^{2}
$$

when the boundary moves with speed $u_{n}$, we come to

$$
\begin{aligned}
& \int_{\Omega(T)} \rho^{f} \frac{|u|^{2}}{2}+\int_{0}^{T} \int_{\Omega(t)} \frac{\mu}{2}\left|\nabla u+\nabla u^{T}\right|^{2}+\int_{\Sigma(T)} \frac{b}{2} \eta^{2} \\
& =\int_{\Omega(0)} \rho^{f} \frac{|u|^{2}}{2}+\int_{\Sigma(0)} \frac{b}{2} \eta^{2}-\int_{0}^{T} \int_{\Gamma}\left(p_{\Gamma}+\frac{\rho^{f}}{2}|u|^{2}\right) u_{n}
\end{aligned}
$$

Thus kinetic energy decreases due to viscosity when $\Gamma=\emptyset$.

The curl-curl low Reynolds approximation of (10) consists in finding $[u, p, \eta]$ with $u \times\left. n\right|_{\partial \Omega}=0,\left.\eta\right|_{\Gamma}=0$ and

$$
\begin{aligned}
& \int_{\Omega}\left[\rho^{f} \partial_{t} u \cdot \hat{u}+\mu \nabla \times u \cdot \nabla \times \hat{u}^{T}-p \nabla \cdot \hat{u}-\hat{p} \nabla \cdot u\right] \\
& +\int_{\Sigma} b\left[\eta \hat{u}_{n}+\hat{\eta}\left(u_{n}-\partial_{t} \eta\right)\right]=-\int_{\Gamma} p_{\Gamma} \hat{u}_{n}, \forall \hat{u}, \hat{p}, \hat{\eta}: \hat{u} \times n=0,\left.\hat{\eta}\right|_{\Gamma}=0
\end{aligned}
$$

Following [14] existence and uniqueness can be shown, subject to the regularity hypotheses therein because the bilinear form $[u, \hat{u}] \rightarrow \int_{\Omega} \nabla \times u \cdot \nabla \times \hat{u}$ is strongly elliptic in the appropriate space of curl free vector fields and the bilinear form $[(p, \eta), \hat{u}] \rightarrow-\int_{\Omega} p \nabla \cdot \hat{u}+b \int_{\Sigma} \eta \hat{u}_{n}$ satisfies the inf-sup condition.

5. A Hierarchy of Approximations on Fixed Domains. First note that $\left.\sigma_{n n}\right|_{\Gamma} \approx$ $-p$, because $\mu$ is small and $\nabla u \approx \mathbf{I} \partial_{n} u_{n} \approx 0$ due to $u \times n=0$. Therefore at the moving wall $\Sigma$,

$$
p=b \eta, u_{n}=\partial_{t} \eta \Rightarrow b u_{n}=\partial_{t} p
$$

To simplify notations let us work with the reduced pressure $p / \rho^{f}$. Then with $b \rightarrow$ $b / \rho^{f}(14)$ is unchanged.

Now recall the transpiration condition concept [9]: instead of applying a boundary condition on a wall $\Sigma(t)$ moving normally by $\eta(x, t)$ we shall apply it on a fixed wall $\Sigma$ with a correction factor based on the following:

$$
u(x+\eta \vec{n})=\vec{n} \frac{\partial \eta}{\partial t}(x), x \in \Sigma(t) \Rightarrow u+\eta \frac{\partial u}{\partial n}=\vec{n} \frac{\partial \eta}{\partial t}+o(\eta) x \in \Sigma
$$

If the radius of curvature of $\Sigma$ is large and if $u \times\left. n\right|_{\partial \Omega}=0$, then, as said earlier,

$$
n \cdot \frac{\partial u}{\partial n} \approx \frac{\partial u_{n}}{\partial n} \approx \nabla \cdot u-\frac{\partial u_{s}}{\partial s}-\frac{\partial u_{\tau}}{\partial \tau}=0
$$

implying that the transpiration correction is of higher order.

So instead of (10) a simpler formulation is obtained after discretization in time and on a fixed domain, as follows. 
5.1. A Model with Velocity and Pressure only. : Find $\left[u^{m+1}, p^{m+1}\right]$ with $u^{m+1} \times n=0$ and

$$
\begin{aligned}
& \int_{\Omega}\left[\hat{u} \cdot\left(\frac{u^{m+1}-u^{m}}{\delta t}-u^{m+\frac{1}{2}} \times \nabla \times u^{m}\right)-p^{m+1} \nabla \cdot \hat{u}-\hat{p} \nabla \cdot u^{m+1}\right] \\
& +\int_{\Omega} \frac{\nu}{2}\left(\nabla u^{m+\frac{1}{2}}+\nabla u^{m+\frac{1}{2}}{ }^{T}\right):\left(\nabla \hat{u}+\nabla \hat{u}^{T}\right) \\
& +\int_{\Sigma}\left(u^{m+\frac{1}{2}} b \delta t+p^{m} \vec{n}\right) \cdot \hat{u}=\int_{\Gamma} p_{\Gamma} \hat{u}_{n} \forall \hat{u}, \hat{p} \text { with } \hat{u} \times n=0
\end{aligned}
$$

We have used the identity: $u \cdot \nabla u=-u \times \nabla \times u+\nabla \frac{|u|^{2}}{2}$ and we have removed the last term which was compensated by the moving domain in (10) and which ought to be absent in the fixed domain model to preserve energy. Indeed, letting $\hat{u}=u^{m+\frac{1}{2}}, \hat{p}=-p^{m+1}$ in (15), leads to the following identity:

$$
\begin{aligned}
& \int_{\Omega}\left|u^{m+1}\right|^{2}+\frac{\nu}{2} \delta t \sum_{k \leq m} \int_{\Omega}\left|\nabla u^{k+\frac{1}{2}}+\nabla u^{k+\frac{1^{2}}{T}}\right|^{2}+b \delta t^{2} \sum_{k \leq m} \int_{\partial \Omega}\left|u^{k+\frac{1}{2}}\right|^{2} \\
& +\frac{1}{2 b} \int_{\partial \Omega}\left[\sum_{k \leq m}\left(p^{k+1}-p^{k}\right)^{2}-p^{m+1^{2}}+p^{0^{2}}\right]=\int_{\Omega}\left\|u^{0}\right\|^{2}
\end{aligned}
$$

This is because an integration by parts of $-p^{m+1} \nabla \cdot \hat{u}$ gives the boundary term $-\int_{\Sigma} p^{m+1} \hat{u}_{n}$ and gathering all integrals on $\Sigma$ in (15) leads to $u^{m+\frac{1}{2}} b \delta t+p^{m} \vec{n}-$ $p^{m+1} \vec{n}=0$.

5.2. A Model Involving only the Pressure. In strong form (15) is

$$
\begin{aligned}
& \partial_{t} u-u \times \nabla \times u+\nabla p-\nu \Delta u=0, \nabla \cdot u=0, \\
& \left.u\right|_{t=0}=u^{0} \text { in } \Omega, \\
& b \vec{u}=\left.\vec{n} \partial_{t} p\right|_{\Sigma} \text { or } \vec{u} \times\left.\vec{n}\right|_{\Gamma}=0,\left.p\right|_{\Gamma}=p_{\Gamma}
\end{aligned}
$$

Taking the divergence of the PDE and its scalar product with $n$ leads to

$$
\begin{aligned}
& -\Delta p=-\nabla \cdot(u \times \nabla \times u) \text { in } \Omega, \\
& \left.\frac{\partial p}{\partial n}\right|_{\Sigma}=\nu \Delta u \cdot n-\partial_{t} u \cdot n=\nu \Delta u \cdot n-\frac{1}{b} \partial_{t t} p,\left.p\right|_{\Gamma}=p_{\Gamma}
\end{aligned}
$$

Note that it could be found also from (15) by choosing $\hat{u}=\nabla q$ and $\hat{p}=0$.

When $u \times \nabla \times u$ and $\left.\nu \Delta u \cdot n\right|_{\Sigma}$ are small, an autonomous equation for $p$ is

$$
-\Delta p=0 \text { in } \Omega, \partial_{t t} p+b \frac{\partial p}{\partial n}=0 \text { on } \Sigma, \Rightarrow \partial_{t t} p_{\Sigma}-b \Delta_{\Sigma} p_{\Sigma}=0
$$

where $-\Delta_{\Sigma}$ is the Steklov-Poincaré operator: $\left.\left.p\right|_{\Gamma} \rightarrow \frac{\partial p}{\partial n}\right|_{\Gamma}$ via $\Delta p=0$.

Two comments:

1. While $\left.\nu \Delta u \cdot n\right|_{\Sigma}$ is generally small for aortic flow, $u \times \nabla \times u$ may not be; except if the flow is irrotational, which is the case only if the flow is nearly the Poiseuille pipe flow. So (19) is clearly only a first approximation to the problem.

2. Resonance may occur in (19) at $\sqrt{b \lambda_{\left(-\Delta_{\Sigma}\right)}}$; this is an important observation which could explain why some computations for the full problem are nearly unstable. 
Resonance is due to the finite length of the aortic geometry used in the computation. To estimate the resonance frequency, is easy in $2 \mathrm{~d}$ : take a rectangle $(0,5) \times(-1,1)$ and the data in (4).

If $p(x, y)=f(x) g(y)$ then $-\Delta p=0$ leads to $f "+a^{2} f=0, g^{\prime \prime}+a^{2} g=0$, as $g$ is maximum at $y=0, g=\cos (a y)$ and so $g^{\prime}(R)=-a \sin (a R)$.

Similarly as $f(0)=f(L)=0, f=\sin (a x)$ with the necessary condition that $a L=k \pi, k \in N^{+}$. So the eigenvalue problem is

$0=\lambda^{2} p(x, R)+b \partial_{y} p(x, R)=\lambda^{2}-b a \sin (a R) \Rightarrow \lambda=\sqrt{b \frac{k \pi}{L} \sin \left(\frac{k \pi}{L} R\right)} \approx \frac{k \pi}{L} \sqrt{b R}(20)$

The first eigenvalue is at $\lambda_{1}=0.9 \mathrm{~cm}$.

6. Discretization with a Finite Element Method. Let $T_{h}$ be a triangulation with $K$ tetraedras $\left\{T_{k}\right\}_{1}^{K}$ with the usual conformity hypotheses; let $\Omega:=\cup_{k} T_{k} \subset$ $\mathbb{R}^{3}$.

Consider the $P^{2}-P^{1}$ element built from

$$
\begin{aligned}
& V_{h}=\left\{v \in C^{0}(\bar{\Omega})^{3}:\left.v_{i}\right|_{T_{k}} \in P^{2}, i=1,2,3\right\} \\
& Q_{h}=\left\{q \in C^{0}(\bar{\Omega}):\left.q\right|_{T_{k}} \in P^{1}\right\}
\end{aligned}
$$

6.1. Discretization of the Full Problem (10). A feasible discretization of (10) is to find $\left[u^{m+1}, p^{m+1}, \eta^{m+1}\right] \in V_{h} \times Q_{h} \times Q_{h}$ with $u^{m+1} \times\left. n\right|_{\Gamma}=0,\left.\eta^{m+1}\right|_{\Gamma}=0$ and such that

$$
\begin{aligned}
& \int_{\Omega}\left[\hat{u} \cdot\left(\frac{u^{m+1}-u^{m}}{\delta t}-u^{m+\frac{1}{2}} \times \nabla \times u^{m}\right)-p^{m+1} \nabla \cdot \hat{u}-\hat{p} \nabla \cdot u^{m+\frac{1}{2}}\right] \\
& +\int_{\Omega}\left[\frac{\nu}{2}\left(\nabla u^{m+\frac{1}{2}}+\nabla u^{m+\frac{1}{2}}{ }^{T}\right):\left(\nabla \hat{u}+\nabla \hat{u}^{T}\right)+\varepsilon \nabla \eta^{m+\frac{1}{2}} \cdot \nabla \hat{\eta}\right] \\
& +\int_{\Sigma} b\left[\eta^{m+\frac{1}{2}} \hat{u}_{n}-\hat{\eta}\left(u_{n}^{m+\frac{1}{2}}-\frac{1}{\delta t}\left(\eta^{m+1}-\eta^{m}\right)\right)\right]=-\int_{\Gamma} p_{\Gamma} \hat{u}_{n} \\
& \forall[\hat{u}, \hat{p}, \hat{\eta}] \in V_{h} \times Q_{h} \times Q_{h} \text { with } \hat{u} \times\left. n\right|_{\partial \Omega}=0,\left.\hat{\eta}\right|_{\Gamma}=0 .
\end{aligned}
$$

where $\varepsilon$ is any small positive parameter.

When $\Omega$ is kept fixed, an energy conservation identity is found by choosing $\hat{u}=$ $u^{m+\frac{1}{2}}, \hat{p}=-p^{m+1}, \hat{\eta}=\eta^{m+\frac{1}{2}}$ :

$$
\begin{aligned}
\int_{\Omega}\left[\frac{u^{m+1^{2}}-u^{m 2}}{\delta t}+\frac{\nu}{2}\left|\nabla u^{m+\frac{1}{2}}+\nabla u^{m+\frac{1}{2}}\right|^{2}+\varepsilon\left|\nabla \eta^{m+\frac{1}{2}}\right|^{2}\right] & +\int_{\Sigma} \frac{\eta^{m+1^{2}}-\eta^{m 2}}{\delta t} \\
& =-\int_{\Gamma} p_{\Gamma} \hat{u}_{n}^{m+\frac{1}{2}}(23)
\end{aligned}
$$

As for the Navier-Stokes equations, when $\delta t$ is small enough the problem has a unique solution because of the energy estimate and because of a general inf-sup condition is satisfied with $p$ replaced by $[p, \eta]$.

6.2. Discretization of Problem (15) in $[u, p]$. A feasible discretization of (15) is to find $u^{m+1} \in V_{h}, p^{m+1} \in Q_{h}$ with $u^{m+1} \times\left. n\right|_{\Gamma}=0$ and such that

$$
\begin{aligned}
& \int_{\Omega}\left[\hat{u} \cdot\left(\frac{u^{m+1}-u^{m}}{\delta t}-u^{m+\frac{1}{2}} \times \nabla \times u^{m}\right)-p^{m+1} \nabla \cdot \hat{u}-\hat{p} \nabla \cdot u^{m+\frac{1}{2}}\right] \\
& +\int_{\Omega} \frac{\nu}{2}\left(\nabla u^{m+\frac{1}{2}}+\nabla u^{m+\frac{1}{2}}{ }^{T}\right):\left(\nabla \hat{u}+\nabla \hat{u}^{T}\right) \\
& +\int_{\Sigma}\left(u^{m+\frac{1}{2}} b \delta t+p^{m} \vec{n}\right) \cdot \hat{u}=-\int_{\Gamma} p_{\Gamma} \hat{u}_{n} \\
& \forall \hat{u} \in V_{h}, \hat{p} \in Q_{h} \text { with } \hat{u} \times\left. n\right|_{\Gamma}=0 .
\end{aligned}
$$


Notice that $u^{m+1} \times\left. n\right|_{\Sigma}=0$ is implied by the formulation. When $\Gamma$ is flat and perpendicular to an axis, that condition amounts to some component of the velocity being zero; it is easy to implement. If not, then it must be added by penalty in the variational formulation.

The difference with a standard Stokes problem is only the added integral on $\Sigma$ which reinforce the ellipticity of the bilinear form. Thanks to the inf-sup condition, the problem is well posed. Error estimates however needs to be rederived.

Notice that the energy equality does not imply stability:

$$
\begin{aligned}
\int_{\Omega}\left[\frac{u^{m+1^{2}}-u^{m 2}}{\delta t}\right. & \left.+\frac{\nu}{2}\left|\nabla u^{m+\frac{1}{2}}+\nabla u^{m+\frac{1}{2}}\right|^{2}\right] \\
& +\int_{\Sigma}\left[b\left|u^{m+\frac{1}{2}}\right|^{2} \delta t+p^{m} u_{n}^{m+\frac{1}{2}}\right]=-\int_{\Gamma} p_{\Gamma} \hat{u}_{n}^{m+\frac{1}{2}}
\end{aligned}
$$

6.3. Stability. For high Reynolds number some upwinding must be applied. Then it is easier to revert to $u \cdot \nabla u$ for the nonlinear term and to use the Characteristic Galerkin method (see [30] and [33] for a second order version). At each time steps one seeks for $u^{m+1} \in V_{h}, p^{m+1} \in Q_{h}$ with $u^{m+1} \times\left. n\right|_{\Gamma}=0$ and

$$
\begin{aligned}
& \int_{\Omega}\left[\hat{u} \cdot\left(\frac{u^{m+1}-u^{m} \circ X^{m}}{\delta t}\right)-p^{m+1} \nabla \cdot \hat{u}-\hat{p} \nabla \cdot u^{m+1}\right] \\
& +\int_{\Omega} \frac{\nu}{2}\left(\nabla u^{m+1}+\nabla u^{m+1}\right):\left(\nabla \hat{u}+\nabla \hat{u}^{T}\right)+b \delta t \int_{\Sigma} u^{m+1} \cdot \hat{u} \\
& =\int_{\Gamma} p_{\Gamma} \hat{u}_{n}-\int_{\Sigma} p^{m} \hat{u}_{n} \forall \hat{u} \in V_{h}, \hat{p} \in Q_{h} \text { with } \hat{u} \times\left. n\right|_{\Gamma}=0
\end{aligned}
$$

where $X^{m}(x)$ is a first order volume preserving (because $\nabla \cdot u^{m} \approx 0$ ), approximation of the solution at $t^{m}$ of

$$
\dot{X}(\tau)=u^{m}(X(\tau), \tau), X\left(t^{m+1}\right)=x .
$$

To be equivalent to (24) an integral of $u^{m+1}(\nabla u) \cdot \hat{u}$ should be added to (26), but we have seen that this term is lost when we passed from a varying domain to a fixed domain. To preserve energy we suggest to drop the term, which means that the formulation is valid approximately only and only if $\left.u^{m}\right|_{\Sigma}$ is small.

Indeed, assuming no quadrature error and choosing $\hat{u}=u^{m+1}, \hat{p}=-p^{m+1}$ leads to

$$
\begin{aligned}
& \frac{1}{\delta t}\left\|u^{m+1}\right\|_{0}^{2}+\nu\left\|\nabla u^{m+1}+\nabla u^{m+1^{T}}\right\|_{0}^{2}+b \delta t\left\|u^{m+1}\right\|_{0, \Sigma}^{2}=\frac{1}{\delta t} \int_{\Omega} u^{m+1} \cdot u^{m} \circ X^{m} \\
& +\int_{\Gamma} p_{\Gamma} u^{m+1}-\int_{\Sigma} p^{m} u_{n}^{m+1}
\end{aligned}
$$

But again the last term seems hard to bound.

A similar use of the Characteristic-Galerkin method can be applied to (24) and there stability is not a problem, so though more expensive, (24) is mathematically a better formulation.

6.4. Discretization of (15) with operator decomposition. We can make explicit use of the boundary condition on the pressure and make a Chorin-like decomposition of (15). The following problems are solved in sequence:

$$
\begin{aligned}
& -\Delta p^{m+1}=\nabla \cdot\left(u^{m} \circ X^{m}\right) \text { in } \Omega \\
& \frac{1}{\delta t^{2}}\left(p^{m+1}-2 p^{m}+p^{m-1}\right)+b \frac{\partial p^{m+1}}{\partial n}=0 \text { on } \Sigma,\left.p^{m+1}\right|_{\Gamma}=p_{\Gamma}
\end{aligned}
$$




$$
\begin{aligned}
& \frac{1}{\delta t}\left(u^{m+1}-u^{m} \circ X^{m}\right)+\nabla p^{m+1}-\nu \Delta u^{m+1}=0 \text { in } \Omega, \\
& \left.u^{m+1}\right|_{\Sigma}=\frac{\vec{n}}{b \delta t}\left(p^{m+1}-p^{m}\right), u^{m+1} \times\left. n\right|_{\Gamma}=0
\end{aligned}
$$

Parallel implementations could take advantage of such decomposition ; however it is not clear that an iterative solution of the full system by block decomposition (see $[18,6])$ is not faster that this Chorin-like decomposition. Second order decomposition should also be studied in this context[31, 21].

6.5. Discretization of the Pressure Problem (19). Alternatively no such difficulty arise with the pressure equation (19); the following scheme, for instance, is stable with a fixed geometry:

$$
\begin{aligned}
& \frac{1}{2} \int_{\Omega} \nabla\left(p^{m+1}+p^{m-1}\right) \cdot \nabla \hat{p}+\frac{1}{b} \int_{\Sigma} \hat{p} \frac{p^{m+1}-2 p^{m}+p^{m-1}}{\delta t^{2}}=0, \\
& \forall \hat{p} \in Q_{h},\left.\hat{p}\right|_{\Gamma}=0, p^{m+1} \in Q_{h},\left.p^{m+1}\right|_{\Gamma}=p_{\Gamma}
\end{aligned}
$$

6.6. Moving the Geometry for Graphic Visualization. The theory requires that $\Sigma$ be moved at every time step along its normal of a quantity $\delta t u^{m} \cdot \vec{n}$. To preserve the triangulation we follow the literature [9] and solve an additional problem

$$
-\Delta \vec{d}^{m+1}=0 \text { in } \Omega,\left.\vec{d}^{m+1}\right|_{\Sigma}=\vec{d}^{m}+\vec{n} \delta t u_{n}^{m},\left.\vec{d}^{m+1}\right|_{\Gamma}=0
$$

and then move every vertex $q^{j}$ of the triangulation $q^{j} \rightarrow q^{j}+\kappa d$. In theory $\kappa=1$ but for graphic enhancement it can be adjusted. Note however that (31) is expensive to solve.

\section{Numerical Tests.}

7.1. Comparison of the Four Methods. We begin with a comparison of (22), $(26),(29)$ and (30) on a simple geometry: a quarter of a torus with a pressure drop imposed from the top horizontal cross section to the right vertical one. The geometry is not updated but moved for graphic rendering.

The cross section of the torus is a circle of radius $1 \mathrm{~cm}$. This circle is extruded on a greater circle of radius $4 \mathrm{~cm}$. The pressure drop is $6 \cos (\pi t), b=200$ and $\nu=0.001$. The time step is 0.05 , voluntarily large to illustrate stability. The computation is stopped at $t=0.75$.

Figure 1 shows the pressure at $t=0.75$ by the four methods. It is seen that (22) and (26) give the same results. However while (30) and (29) agree with each other (an indication that $u \times \nabla \times u$ is small) but only qualitatively with (22). The computing time is given in Table 1 The linear s are solved with the library UMFPACK which

TABle 1. Computing time for (22), (26),(29) and (30)

\begin{tabular}{|c||c|c|c|c|}
\hline Method & {$[u, v, w, p, \eta]$} & {$[u, v, w, p]$} & $p \rightarrow[u, v, w]$ & $p$ \\
\hline CPU & 10.9 & 9.6 & 31.5 & 5.38 \\
\hline
\end{tabular}

explains why (29) is so much more expensive. The mesh has 1395 vertices giving 6975 degrees of freedom for each linear systems for $\left[u^{m+1}, v^{m+1}, w^{m+1}, p^{m+1}, \eta^{m+1}\right]$ . Figure 2 shows the values of $|u \times \times u|$ and $\nu n^{T}(\nabla u) n$ when the flow is computed 
$\mathrm{t}=0.75$

$t=0$
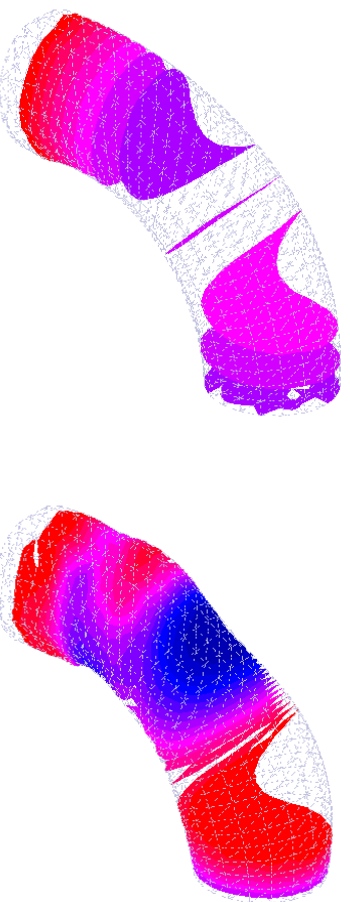
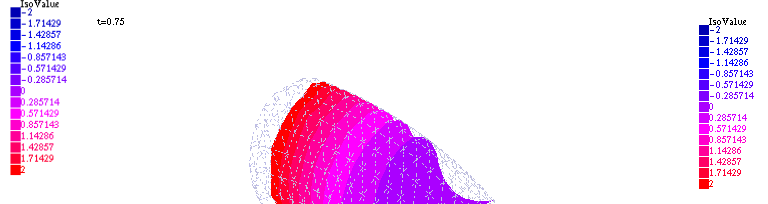

$t=0.75$
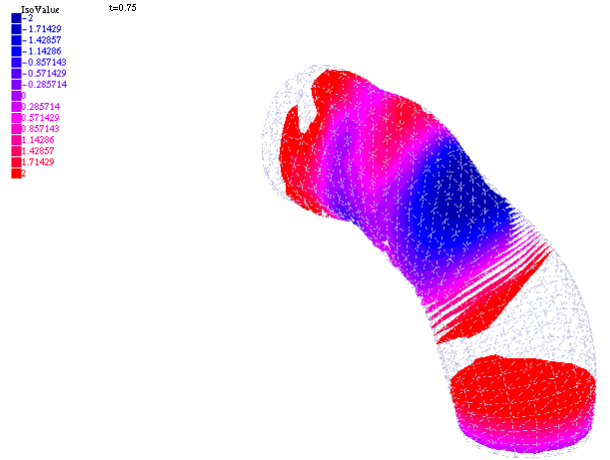

Figure 1. Top left: computation of $[u, v, w, p, \eta]$ with (22). Top right: computation of $[u, v, w, p]$ with $(26)$. Bottom left: computation of $[u, v, w, p]$ using operator decomposition (29). Bottom right: computation of the pressure only $p$ with (30).

using the $[u, v, w, p]$ model. It is seen that $\nu n^{T}(\nabla u) n$ is not small at the lower section while $\nu n^{T}(\nabla u) n$ is small everywhere.

7.2. Performance for the Aorta. The geometry is a section of the aorta obtained from a MRI scan. It has 4991 vertices, giving 19964 degrees of freedom for each linear systems for $\left[u_{1}^{m+1}, u_{2}^{m+1}, u_{3}^{m+1}, p^{m+1}\right]$. The pressure drop from inflow section on the right to outflow section on the left is $p_{\Gamma_{R}}=6 \cos ^{2}(\pi t)$ and the results are shown at $t=0.8$. On the smaller cross sections a pressure drop equal to $p_{\Gamma_{R}} / 2$ is imposed. Model (26) is used with $\delta t=0.05, \nu=0.001, b=200$. The computation took 342 " on a macbook pro 15 ", $2012,2.3 \mathrm{MHz}$ core i7.

\subsection{Performance on a Documented Stenosed Carotid Bifurcation Flow.}

In this section we reproduce the computational results of pulsatile flow in human carotid bifurcation models $[19,20]$. The referred studies assumed rigid wall arteries. We further extend those results to the full fluid-structure interaction problem with compliant walls.

We have modeled a $10 \mathrm{~cm}$ long representative healthy carotid artery bifurcation model, along with $30 \%$ and $64 \%$ concentrically stenosed models. The common 


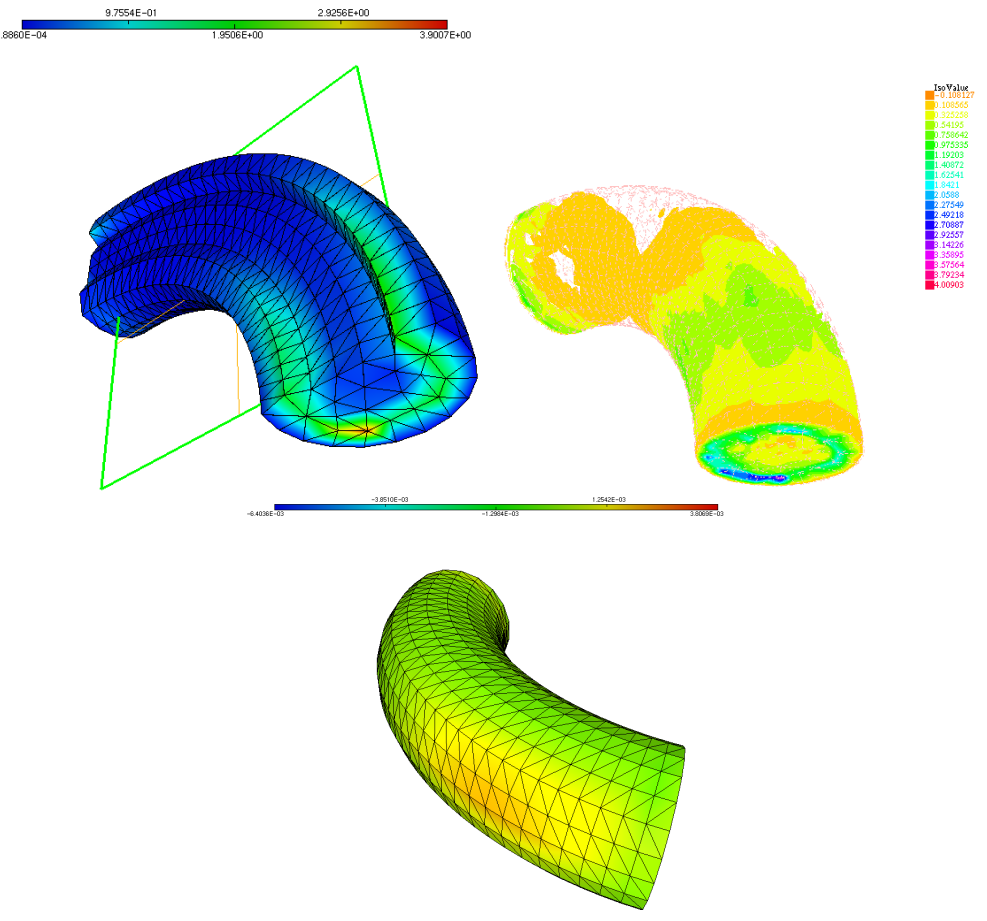

Figure 2. Top: two views of $|u \times \nabla \times u|$. Bottom: $\nu n^{T}(\nabla u) n$ on the wall of the vessel. The lower section of the tube faces the reader.
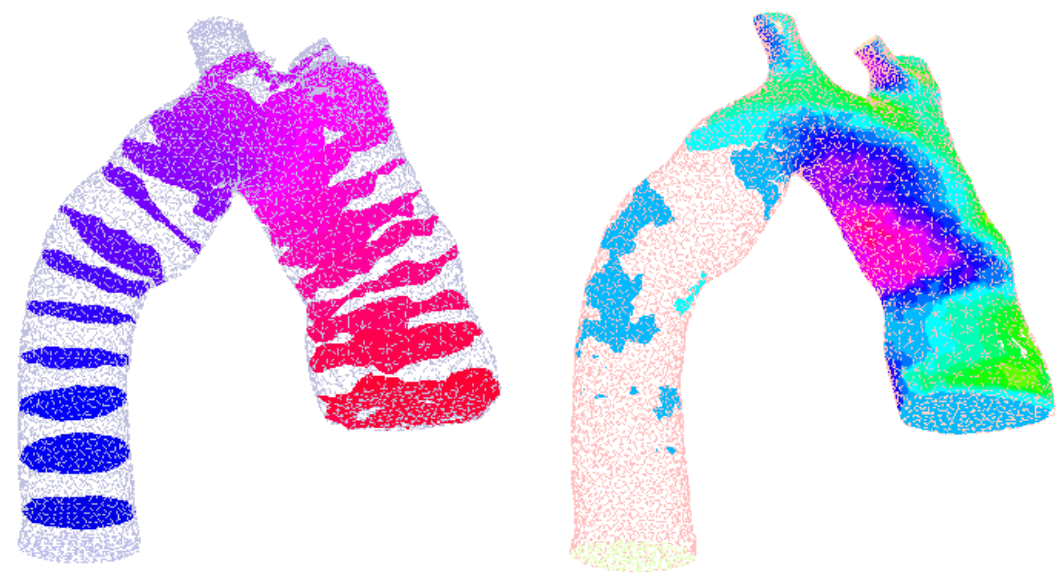

Figure 3. Computation of $[\vec{u}, p]$ with (26) for an aorta. Left: pressure at $t=0.8$. The compliant walls are updated for graphic visualization of the compliant wall deformation. On the Left the vertical velocity $u_{3}$ at $t=0.8$ without graphic enhancement, thus showing also the geometry used for the computation. 
carotid artery has a diameter of $9 \mathrm{~mm}$, the internal and external carotid arteries have a diameter of $6.8 \mathrm{~mm}$ and $5.6 \mathrm{~mm}$, respectively. All artificial boundaries are perpendicular to $x$ - axis. Finite element meshes (coarse discretization $h=0.2 \mathrm{~mm}$, 1885 vertices) are shown in Figure 4. Fine discretizations are built with $h=0.04 \mathrm{~mm}$ resulting roughly in $2 \cdot 10^{5}$ vertices and one million tetrahedral elements.

First, in order to compare our results with those cited above, we consider $\Sigma$ to be a rigid wall. We also replaced the inlet boundary condition $\int_{\Gamma} p_{\Gamma} \hat{u}_{n}$ by the velocity profile

$$
U_{\Gamma}=2 Q / A_{C C A}\left(1-y^{2} / R_{C C A}^{2}-z^{2} / R_{C C A}^{2}\right)
$$

where $Q$ is the flow rate depicted in Figure 4 (right). Spline interpolation was used to fit the pointwise data; traction-free boundary conditions were imposed at the outlet boundaries.

The initial velocity-pressure field at $t=0$ is set to be a solution of the Oseen equation. The time step is 0.01 . Five cardiac cycles are sufficient to eliminate transient effects caused by initial conditions, and to obtain cyclically repeated flow patterns. For the considered geometries, the inlet flow together with $\nu=3.5 \cdot 10^{-3} \mathrm{~Pa} \cdot \mathrm{sec}$ and $\rho=1060 \mathrm{~kg} \cdot \mathrm{m}^{-3}$ result in a Reynolds number of a few hundreds; Re at the inflow surface is $357, R e$ at the $64 \%$ stenosed area is 1280 . Excellent agreement with the published results was observed in booth magnitude and location of flow patterns.
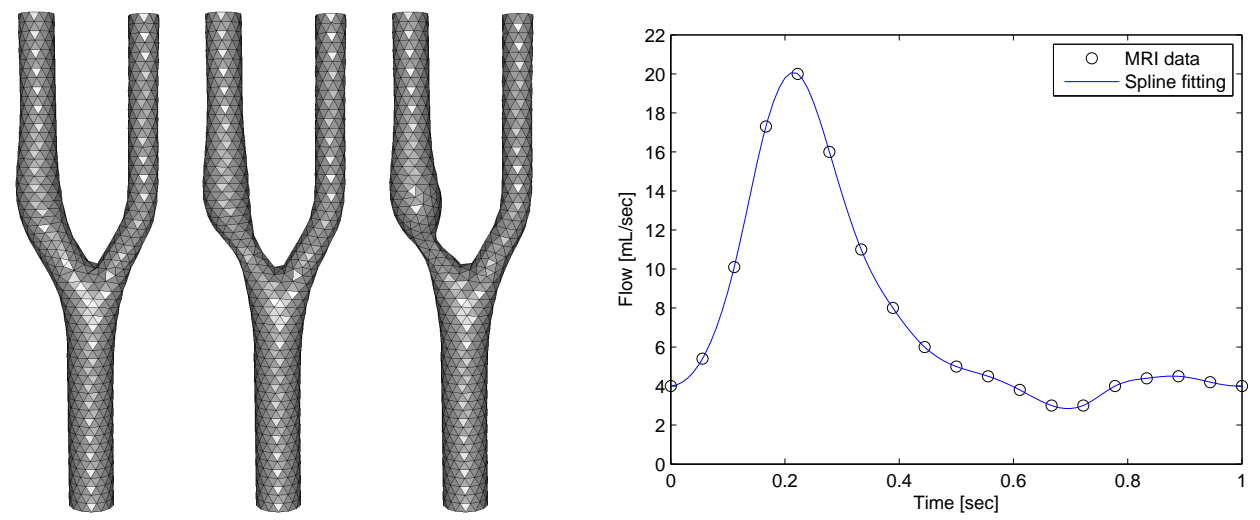

Figure 4. Carotid bifurcation: healthy (left), 30\% (middle), $64 \%$ (right) stenosed models (coarse discretization). Flow rate waveform at the common carotid artery inlet.

Now we use model (26) with $b=200$ and $\delta t=0.01$. The geometry is fixed for the numerical simulations but updated for graphic rendering, $\kappa=100$. We observe that for all three models the maximum positive displacement, i.e. expansion $(\vec{d} \cdot \vec{n}>0)$ is at $t=0.17$, while the maximum negative displacement i.e. contraction is at $t=0.64$. Figure 5 shows the updated shape of the bifurcation region at four selected phases of the cardiac cycle: $t=0, t=0.1, t=0.15$ and $t=0.17$. The red color marks regions with higher pressure. We observe that the healthy artery undergoes a uniform expansion/contraction in the entire domain. For the stenosed arteries, displacements become much larger in the portion of the internal carotid artery below the bifurcation; we identify tiny displacements downstream from the stenosis. More importantly, a steep change in pressure near the stenosis region 
observed for both $30 \%$ and $64 \%$ stenosis models will produce an increasing shear stress that could be a reason for arterial rupture.
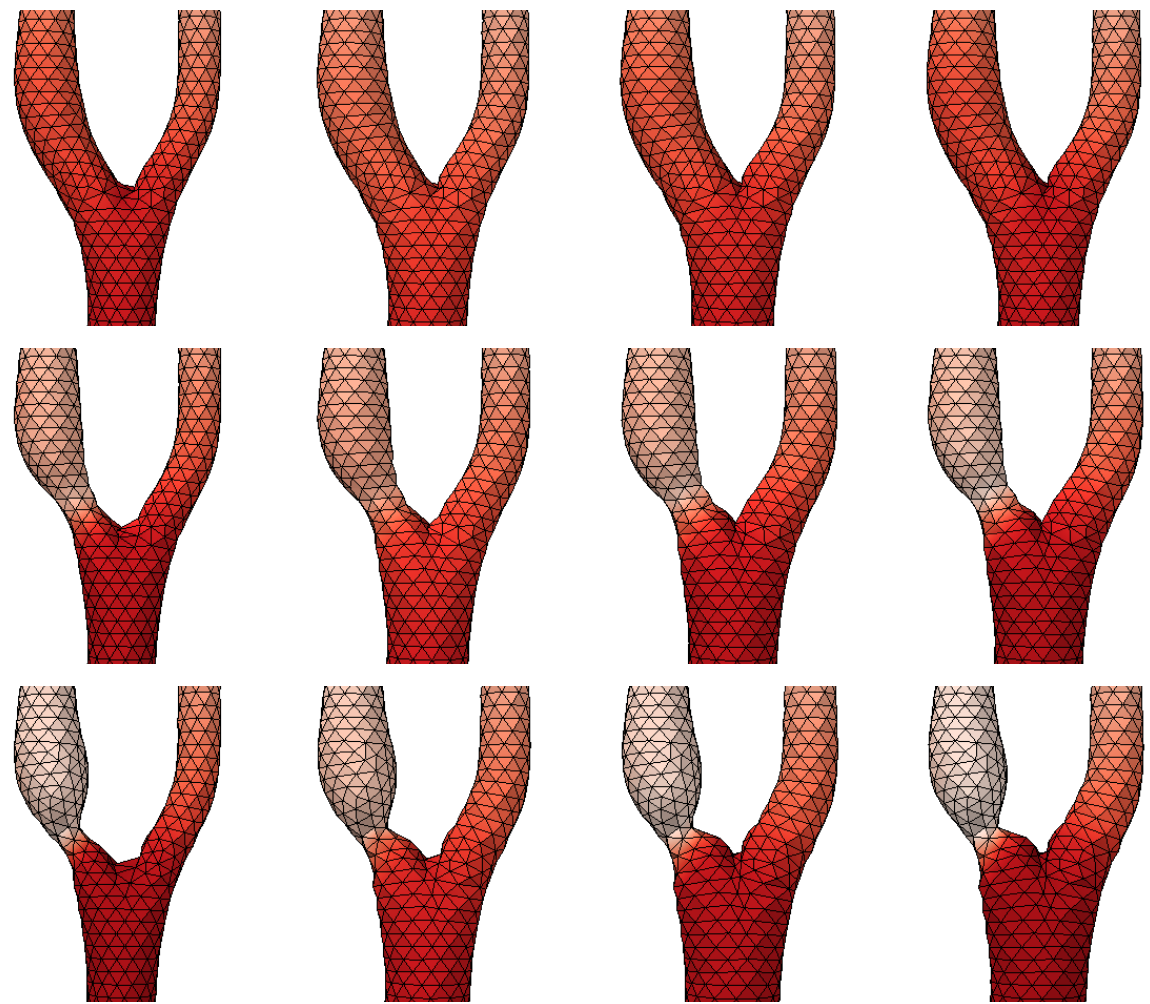

$t=0.00$

$t=0.10$

$t=0.15$

$t=0.17$

Figure 5. Carotid bifurcation. Arterial displacement during the pre-systolic period; top to bottom: normal, 30\%, 64\% stenosis. Dark red color marks regions of higher pressure.

The present study has demonstrated that model (26) allows physiologically realistic blood flow analysis. A hierarchy of approximations helps reducing the overall computational effort of updating the triangulation at every time step. On the other hand, the method allows to inspect arterial compliance at arbitrary phases of the cardiac cycle. More importantly, moving the geometry for graphic visualization can be done as a post-processing task, or in parallel.

\section{REFERENCES}

[1] C. Begue, C. Conca, F. Murat, and O. Pironneau. Les equations de Stokes et de NavierStokes avec condtions aux limites sur la pression, pp. 179-264. Pittman, RNM Longman, 1988. In H. Brezis, J. Lions (eds): Nonlinear Partial Differential Equations and their applications, College de France, Vol. IX. Pitman, Boston.

[2] D. Boffi, L. Gastaldi, A FEM for the immersed boundary method, Comput. Struct. 81(2003),491-501.

[3] M. Costabel and M. Dauge, Maxwell and Lamé Eigenvalues on Polyhedral Domains. Preprint Université de Rennes I, 2002. 
[4] M. Costabel, M. Dauge. Singularities of electromagnetic fields in polyhedral domains. Preprint 97-19, Université de Rennes 1 (1997). http://www.maths.univ-rennes1.fr/ dauge/.

[5] G.H. Cottet, E. Maitre and T. Milcent. Eulerian Formulation and Level Set Models For Incompressible Fluid-Structure Interaction. ESAIM: M2AN 42 (2008) 471-492.

[6] P. Crosetto, S. Deparis, G. Fourestey, A. Quarteroni Parallel Algorithms For FluidStructure Interaction Problems In Haemodynamics. SIAM J. SCI. COMPUT. Vol. 33, No. 4, pp. 1598-1622 (2011).

[7] A. Decoene \& B. Maury Moving Meshes with freefem++. J. Numer Math (20)3-4, p195$214(2013)$.

[8] J. De Hart, G. Peters, P. Schreurs and F. BaAijens. A three-dimensional computational analysis of fluid-structure interaction in the aortic valve. J. Biomechanics 2003; 36:103-112.

[9] S. Deparis, M.A. Fernandez and L. Formaggia, Acceleration of a Fixed Point Algorithm for Fluid-Structure Interaction using Transpiration Conditions. ESAIM:M2AN Vol.37,No 4,2003, 601-616.

[10] Â FAng Q. Hu, X.D. LI, D.K. Lin. Absorbing boundary conditions for nonlinear Euler and Navier-Stokes equations based on the perfectly matched layer technique. J. of Comp. Physics 227 (2008) 4398-4424.

[11] M. Fernandez, Incremental displacement-correction schemes for incompressible fluidstructure interaction. Numer. Math. (2013) 123:21-65.

[12] L. Formaggia, J.F. Gerbeau, F. Nobile, A. Quarteroni, On the coupling of 3D and 1D Navier-Stokes equations for flow problems in compliant vessels. Comput. Methods Appl. Mech.Engrg.191(2001)561-582.

[13] L. Formaggia, A. Quarteroni, A. Veneziani eds. Cardiovasuclar Mathematics. Springer MS\&A series 2009.

[14] V. Girault, R. Glowinski, Error analysis of a fictitious domain method applied to a Dirichlet problem, Japan Journal of Ind. and Applied Math., 12, 3, 487-514 (1995).

[15] V.Girault,P.A.Raviart, Finite Element Method for Navier-Stokes Equations.SCM 5. Berlin, Heidelberg, NewYork: Springer 1986.

[16] Â O. Gonzalez, J.C. Simo On the Stability of Symplectic and Energy-Momentum Algorithms for Nonlinear Hamiltonian Systems with Symmetry. Comput. Methods Appl. Mech. Engrg. 134 (1996) 197-222.

[17] O. Gonzalez Exact energy and momentum conserving algorithms for general models in nonlinear elasticity. Comput. Methods Appl. Mech. Engrg. 190 (2000) 1763-1783.

[18] K. Gostaf, O.Pironneau , and F.X. Roux. Finite Element Analysis of Multi - Component Assemblies: CAD - based Domain Decomposition. DDM 14 Proc. (to appear).

[19] I. Marshall. Computational Simulations and Experimental Studies of 3D Phase-Contrast Imaging of Fluid Flow in Carotid Bifurcation Geometries. Journal of Magnetic Resonance Imaging 31 (2010) 928-934.

[20] D.A. Steinman, T.L.Poepping, M. Tambasco, R.N. Rankin, and D.W. Holdsworth. Flow Patterns at the Stenosed Carotid Bifurcation: Effect of Concentric versus Eccentric Stenosis. Annals of Biomedical Engineering 28 (2000) 415-423.

[21] J. L. Guermond, P. Minev, And J. Shen. An overview of projection methods for incompressible flows. Comput. Methods Appl. Mech. Engrg., 195(44-47):6011-6045, 2006.

[22] M. Heil, A. Hazel, J. Boyle. Solvers for large-displacement fluid-structure interaction problems: segregated versus monolithic approaches. Comput Mech (2008) 43:91-101.

[23] P. LE TALLEC Fluid structure interaction with large structural displacements. Comput. Methods Appl. Mech. Engrg 190, 3039-3067. 2001.

[24] F. Nobile and C. Vergana, an effective fluid-structure interaction formulation for vascular dynamics by generalized robin conditions. SIAM J. Sci. Comp. Vol. 30, No. 2, pp. 731-763 (2008).

[25] C. PARes Un traitement faible par élément finis de la condition de glissement sur une paroi pour les équations de Navier-Stokes. C. R. Acad. Sci. Paris Sér. I Math., Vol. 307, pp.101-106, 1988.

[26] C. Peskin, The immersed boundary method, Acta Numerica 11 (2002),479- 517.

[27] C. Peskin and D. McQueen, A three dimensional computational method for blood flow in the hearth-I. Immersed elastic fibers in a viscous incompressible fluid, J. Comput. Phys. 81 (1989) 372-405.

[28] O. Pironneau. Finite Element Methods for Fluids, Wiley 1989. 
[29] O. Pironneau. Conditions aux limites sur la pression pour les équations de Stokes et de Navier-Stokes. C. R. Acad. Sci. Paris Sér. I Math., 303(9):403-406, 1986.

[30] OP and M.TABATA. Stability and convergence of a Galerkin-characteristics finite element scheme of lumped mass type Int. J. Numer. Meth. Fluids 2010; 64:1240-1253.

[31] R. Rannacher, Incompressible Viscous Flow. Encyclopedia Mecanicae. E. Stein ed. Wiley 2004.

[32] J. RAppaz, S. Flotron Numerical conservation schemes for convection-diffusion equations (to appear).

[33] ZhiYONG Si. Second order modified method of characteristics mixed defect-correction finite element method for time dependent Navier-Stokes problems. Numer Algor (2012) 59:271-300.

[34] J. Tambaca, S. Canic, M. Kosor, R.D. Fish, D. Paniagua. Mechanical Behavior of Fully Expanded Commercially Available Endovascular Coronary Stents. Tex Heart Inst J 2011;38(5):491-501).

[35] M. Thiriet, Biomathematical and Biomechanical Modeling of the Circulatory and Ventilatory Systems. Vol 2: Control of Cell Fate in the Circulatory and Ventilatory Systems. Springer Math\& Biological Modeling 2011.

[36] F. Usabiaga, J. Bell, R. Buscalioni, A. Donev, T. Fai, B. Griffith, and C. Peskin. Staggered schemes for fluctuating hydrodynamics. Multiscale Model Sim. 10:1369-1408, 2012.

[37] I. Vignon-Clementel, A. Figueroa, K. Jansen, C.A. Taylor Outflow boundary conditions for three-dimensional finite element modeling of blood flow and pressure in arteries. Comput. Methods Appl. Mech. Engrg. 195 (2006) 3776-3796.

E-mail address: 0livier.Pironneau@upmc.fr \& gostaf@sfu.ca 